$1-1-1998$

\title{
Physical Dependence of the Sensitivity and Room-Temperature Stability of AuxGe1-x Thin Film Resistive Thermometers on Annealing Conditions
}

\author{
Nathanael A. Fortune \\ Smith College, nfortune@smith.edu \\ Michael J. Graf \\ Boston College \\ Keizo Murata \\ Osaka City University
}

Follow this and additional works at: https://scholarworks.smith.edu/phy_facpubs

Part of the Physics Commons

\section{Recommended Citation}

Fortune, Nathanael A.; Graf, Michael J.; and Murata, Keizo, "Physical Dependence of the Sensitivity and Room-Temperature Stability of AuxGe1-x Thin Film Resistive Thermometers on Annealing Conditions" (1998). Physics: Faculty Publications, Smith College, Northampton, MA.

https://scholarworks.smith.edu/phy_facpubs/80

This Article has been accepted for inclusion in Physics: Faculty Publications by an authorized administrator of Smith ScholarWorks. For more information, please contact scholarworks@smith.edu 


\section{Physical dependence of the sensitivity and room-temperature stability of $\mathrm{Au}_{x} \mathrm{Ge}_{1-x}$ thin film resistive thermometers on annealing conditions}

Cite as: Review of Scientific Instruments 69, 133 (1998); https://doi.org/10.1063/1.1148488

Submitted: 22 September 1997 • Accepted: 24 October 1997 • Published Online: 04 June 1998

Nathanael A. Fortune, Michael J. Graf and Keizo Murata

ARTICLES YOU MAY BE INTERESTED IN

High magnetic field corrections to resistance thermometers for low temperature calorimetry

Review of Scientific Instruments 71, 3825 (2000); https://doi.org/10.1063/1.1310341

Heat Capacity Measurements on Small Samples at Low Temperatures

Review of Scientific Instruments 43, 205 (1972); https://doi.org/10.1063/1.1685596

Fabrication of Ge-Au film thermometers using interfacial atomic diffusion

Review of Scientific Instruments 64, 2624 (1993); https://doi.org/10.1063/1.1143880

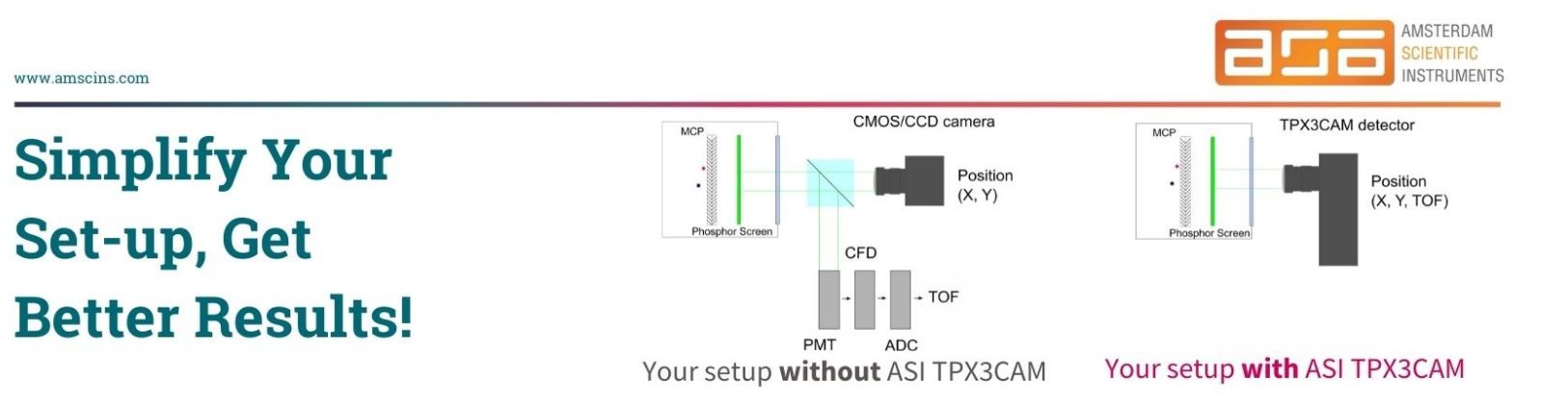

Review of Scientific Instruments 69, 133 (1998); https://doi.org/10.1063/1.1148488 


\title{
Physical dependence of the sensitivity and room-temperature stability of $\mathrm{Au}_{x} \mathrm{Ge}_{1-x}$ thin film resistive thermometers on annealing conditions
}

\author{
Nathanael A. Fortune \\ Department of Physics, Smith College, Northampton, Massachusetts 01063 \\ Michael J. Graf \\ Department of Physics, Boston College, Chestnut Hill, Massachusetts 02167 \\ Keizo Murata \\ Department of Material Science, Faculty of Science, Osaka City University, Sumiyoshi-ku, Osaka 558, \\ Japan
}

(Received 22 September 1997; accepted for publication 24 October 1997)

\begin{abstract}
The reported nearly constant temperature sensitivity of appropriately annealed polycrystalline $\mathrm{Au}_{x} \mathrm{Ge}_{1-x}$ thin films at cryogenic temperatures would appear to make them promising materials for low mass, rapid thermal response resistive thermometers, but their adoption has been limited by difficulties in fabrication and uncertainties in annealing. In this work, we present a method of fabrication and annealing which allows control of the two most important parameters for these films: the room-temperature resistivity $\rho^{\mathrm{RT}}$ and the temperature sensitivity $\eta(T)$, where $\eta$ $\equiv-d \ln R / d \ln T$. We find that the dependence of $\rho^{\mathrm{RT}}$ on total anneal duration $t$ for $x \approx 0.18$ is given by $\rho^{\mathrm{RT}}=\rho_{\infty}[1-A \exp (-t / \tau)]$, where the limiting room-temperature resistivity $\rho_{\infty}$, the annealing coefficient $A$, and relaxation time $\tau$ are annealing temperature dependent parameters. The dependence of $\rho^{\mathrm{RT}}$ and temperature calibration $\rho(T)$ on anneal duration can be minimized by annealing above $250{ }^{\circ} \mathrm{C}$. Like $\rho^{\mathrm{RT}}$, the sensitivity $\eta(T)$ also depends on annealing temperature, with higher annealing temperatures corresponding to lower cryogenic sensitivities. In all cases $\eta(T)$ can be well described by a polynomial expansion in $\ln T$ from room temperature down to at least $2 \mathrm{~K}$. (C) 1998 American Institute of Physics. [S0034-6748(98)05901-2]
\end{abstract}

\section{INTRODUCTION}

The temperature dependent resistivity $\rho(T)$ of polycrystalline $\mathrm{Au}_{x} \mathrm{Ge}_{1-x}$ thin films with $x \approx 0.18$ is sometimes reported to obey the power law temperature dependence

$$
\frac{\rho}{\rho^{*}}=\left(\frac{T}{T^{*}}\right)^{-\eta}
$$

over a wide temperature range, where the temperature sensitivity $\eta$ is a constant that depends on the exact Au concentration and annealing conditions ${ }^{1-3}$ and where $\rho^{*}$ and $T^{*}$ are experimentally determined constants. The prospect of nearly constant sensitivity $\eta$ in a low mass, rapid thermal response thin film makes polycrystalline $\mathrm{Au}_{x} \mathrm{Ge}_{1-x}$ an attractive thermal sensor, particularly for small sample calorimetry. Unfortunately, several difficulties are encountered when fabricating these films by standard methods. ${ }^{1,4,5}$ These difficulties include: (1) a changing Au concentration during evaporation due to the differing evaporation rates $^{2}$ for $\mathrm{Au}$ and $\mathrm{Ge}$, (2) drifts in the room-temperature resistivity, ${ }^{1}$ and (3) the complex dependence of the resistivity on the annealing conditions used to transform the as deposited partially amorphous material into a polycrystalline film. . $^{1,3}$

Some of these difficulties can be overcome using a fabrication method introduced by Zhu and Lin, ${ }^{2}$ in which a series of very thin $\mathrm{Ge}$ and Au layers are sequentially deposited using an electron-beam. Their method is based on the observation that the atoms in bilayers of $\mathrm{Au}$ and $\mathrm{Ge}$ interdiffuse at and above room temperature to produce an amorphous alloy of the desired $\mathrm{Au}$ concentration at the Au/Ge interface. ${ }^{6}$ In principle, an amorphous alloy made from very thin layers could be deposited at room temperature and then be annealed to produce a polycrystalline film with the desired sensitivity. In practice, Zhu and Lin's films were effectively annealed in situ as they were deposited due to radiation heating from the $\mathrm{Au}$ and Ge sources. ${ }^{2}$ Nevertheless, this sequential deposition method allows the production of stable polycrystalline $\mathrm{Au}_{x} \mathrm{Ge}_{1-x}$ films with a nearly constant sensitivity comparable to conventional flash-evaporated films annealed ex situ at $130{ }^{\circ} \mathrm{C} .{ }^{1}$

It should be noted that Zhu and Lin's films exhibited an unexplained dependence of the room temperature resistivity $\rho^{\mathrm{RT}}$ and sensitivity $\eta(T)$ on the thickness of the Au layers, even though the average $\mathrm{Au}$ concentration $x$ remained constant. $^{2}$ This prompted us to wonder if uncontrolled changes in the in situ annealing conditions could be responsible. If so, an understanding of annealing process might allow us to overcome the remaining difficulties in reliably fabricating $\mathrm{Au}_{x} \mathrm{Ge}_{1-x}$ thermometers with negligible drift and controllable sensitivity. In this work, we have modified Zhu and Lin's deposition method to avoid in situ annealing, thereby allowing an investigation of the dependence of the resistive properties of $\mathrm{Ge} / \mathrm{Au}$ multilayers on annealing conditions. We find that the resistive properties can be changed in a controlled manner through annealing and present analytic expressions for the dependence of $\rho^{\mathrm{RT}}$ on annealing temperature and duration and the dependence of $\rho(T)$ and $\eta(T)$ on temperature for fully annealed films. 


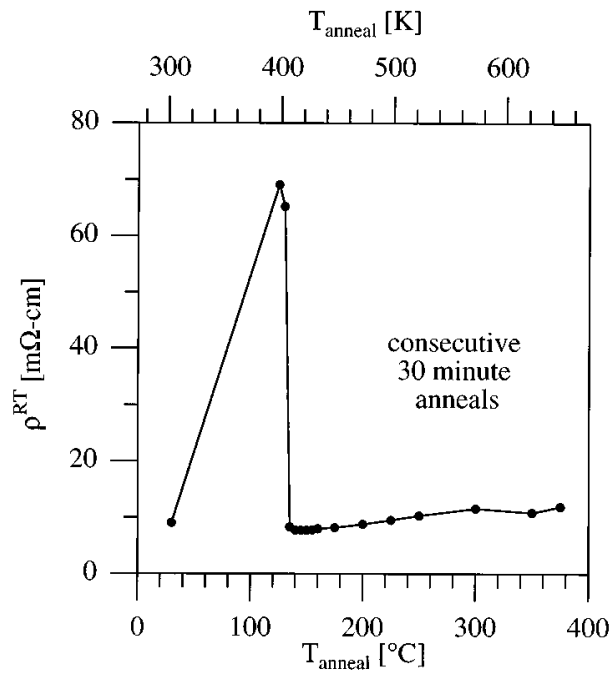

FIG. 1. Dependence of room-temperature resistivity $\rho^{\mathrm{RT}}$ on annealing temperature for an $\mathrm{Au}_{0.18} \mathrm{Ge}_{0.82}$ thin film formed from 50 bilayers of $\mathrm{Au}$ and $\mathrm{Ge}$. The line connecting each data point is a guide to the eye. The Au and Ge layers were deposited by sputtering at $40{ }^{\circ} \mathrm{C}$. After deposition, the film was annealed for 30 minutes at each of a series of increasing temperatures indicated on the graph. The film was allowed to cool to room temperature to measure $\rho^{\mathrm{RT}}$ between each anneal. The sharp drop in $\rho^{\mathrm{RT}}$ after annealing at $135^{\circ} \mathrm{C}$ is due to the onset of Au-assisted Ge crystallization.

\section{EXPERIMENT}

\section{A. Sample preparation}

The films used in this study were prepared by sputtering 50 bilayers of $\mathrm{Ge}$ and Au through a simple shadow mask onto a single crystal sapphire substrate (with previously deposited electrical contacts) in the presence of $30 \mathrm{mTorr}$ of $\mathrm{Kr}$ gas. Each bilayer is composed of $8.4 \AA$ of $A u$ on top of $43 \AA$ of $\mathrm{Ge}$, corresponding to 18 at. \% $\mathrm{Au}$. To avoid annealing the films during deposition, the alternating layers of $\mathrm{Ge}$ and $\mathrm{Au}$ films were sequentially sputtered at $40{ }^{\circ} \mathrm{C}$. Electrical contact to the deposited $\mathrm{Au} / \mathrm{Ge}$ film was made by wire-bonding $\mathrm{Au}-$ $0.5 \% \mathrm{Ga}$ wires to the electrical contacts, which consisted of 1 mm thick sputtered Au contacts on top of $1000 \AA$ thick sputtered $\mathrm{Cr}-7 \% \mathrm{Ti}$ alloy binding layers.

After deposition, the films were removed from the deposition chamber and sequentially annealed at a series of increasing temperatures. The films were allowed to cool down to room temperature between each anneal before measuring the film resistance. All films were exposed to air at room temperature before annealing began and annealing was done in either an air, nitrogen or argon atmosphere according to convenience. No dependence of our results on annealing atmosphere was observed.

\section{B. Annealing process}

As shown in Fig. 1 for a film annealed for $30 \mathrm{~min}$ at each annealing temperature, the room-temperature resistivity increases nearly an order of magnitude after annealing at 125 ${ }^{\circ} \mathrm{C}$, only to sharply drop below its original pre-annealed value after two additional anneals at $130^{\circ} \mathrm{C}$ and $135^{\circ} \mathrm{C}$. A sharp drop in resistivity after annealing originally amorphous $x \approx 0.18 \mathrm{Au}_{x} \mathrm{Ge}_{1-x}$ films has also been seen for co-sputtered ${ }^{3}$ and co-evaporated ${ }^{4}$ thin films. The inferred minimum tem- perature of $135^{\circ} \mathrm{C}$ for crystallization is in good agreement with the $130{ }^{\circ} \mathrm{C}$ value reported for both flash evaporated ${ }^{1}$ and co-sputtered ${ }^{3}$ films.

At still higher annealing temperatures, we see from Fig. 1 that the room-temperature resistivity increases only slowly after further anneals and appears to saturate as the annealing temperature approaches the eutectic temperature $\left(361^{\circ} \mathrm{C}\right)$. Briefly annealing at a temperature slightly above the eutectic temperature $\left(30 \mathrm{~min}\right.$ at $375^{\circ} \mathrm{C}$ ) produced discoloration at the electrical contacts but did not lead to a pronounced change in the resistivity of the film.

The change in the room-temperature resistivity on maximum annealing temperature can be understood as follows. During deposition at $40{ }^{\circ} \mathrm{C}$, the $\mathrm{Au}$ atoms and $\mathrm{Ge}$ atoms partially interdiffuse to form an amorphous alloy of $\mathrm{Au}$ and $\mathrm{Ge}$ at the $\mathrm{Au} / \mathrm{Ge}$ interfaces. In agreement with $\mathrm{x}$-ray absorption fine structure experiments, ${ }^{6}$ we find that the layers do not completely interdiffuse at $40{ }^{\circ} \mathrm{C}$, since the film resistance is shunted if the first layer deposited on top of the electrical contacts is $\mathrm{Au}$ instead of Ge. During anneals at temperatures below $135^{\circ} \mathrm{C}$, the room temperature resistivity increases after each anneal as Au atoms that have diffused into the amorphous Ge layer begin to cluster into Au grains and as more atoms from the $\mathrm{Au}$ and $\mathrm{Ge}$ layers interdiffuse.

During anneals at temperatures at and above $135^{\circ} \mathrm{C}$, however, the relatively weak Au-Ge bonding in the amorphous $x \approx 0.18 \mathrm{Au}_{x} \mathrm{Ge}_{1-x}$ film mediates the exchange of $\mathrm{Ge}$ for $\mathrm{Au}$, allowing the rearrangement and crystallization of $\mathrm{Ge}$ in a process known as metal-assisted crystallization. ${ }^{6-9}$ Due to the vanishingly low solid solubility of $\mathrm{Au}$ in crystalline $\mathrm{Ge}$ at these temperatures, the $\mathrm{Au}$ atoms are swept to the crystallization front, promoting further $\mathrm{Ge}$ crystallization and $\mathrm{Au}$ expulsion. ${ }^{6,9}$ Ultimately, all the Au atoms in the previously amorphous alloy are confined to polycrystalline Ge grain boundaries, significantly restricting further Au diffusion for low Au concentrations. ${ }^{6}$ The sharp drop in resistivity at the onset of Au-assisted crystallization of $\mathrm{Ge}$ is attributed to the changes in system volume, ${ }^{4}$ which increases the electron density.

It is important to note that the critical temperature for metal-assisted crystallization is concentration dependent. For small metal concentrations, the critical temperature at which metal-assisted crystallization occurs decreases with increasing metal concentration until reaching a temperature on the order of $2 / 3$ of the eutectic temperature, ${ }^{7}$ which may be the

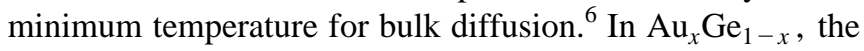
critical temperature drops from $330{ }^{\circ} \mathrm{C}$ to $130^{\circ} \mathrm{C}$ as the $\mathrm{Au}$ concentration increases from $x=0.04$ to $x=0.22 .^{6}$

The measurements shown in Fig. 1 are necessary to identify a minimum annealing temperature but do not tell us if we have performed a sufficiently long anneal to fully crystallize the as-deposited partially amorphous material. In addition, metal-assisted crystallization provides an explanation of the sharp drop in resistivity at $135^{\circ} \mathrm{C}$, but does not readily explain the slow rise in room-temperature resistivity at still higher annealing temperatures, once Ge crystallization has occurred.

To answer these questions, we now turn to our results for the change in the room-temperature resistivity $\rho^{\mathrm{RT}}$ with 


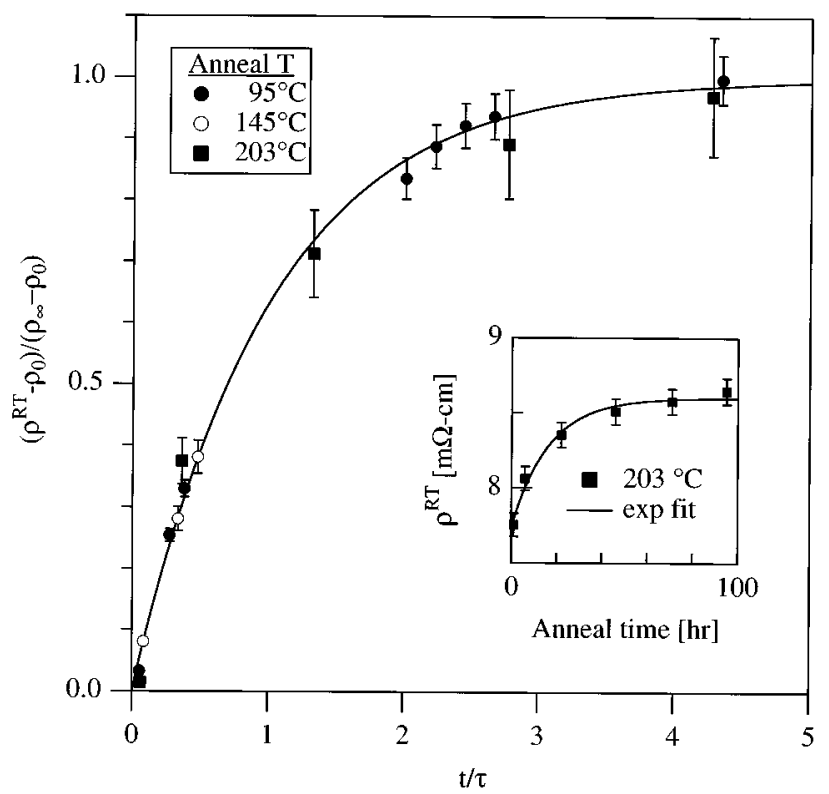

FIG. 2. Dependence of the room-temperature resistivity $\rho^{\mathrm{RT}}$ on annealing duration (at a fixed annealing temperature) for three different annealing temperatures. The data are plotted in terms of the fractional change in the room-temperature resistivity $\left(\rho^{\mathrm{RT}}-\rho_{0}\right) /\left(\rho_{\infty}-\rho_{0}\right)$ and the relative anneal duration $t / \tau$, where the fitting parameters $\rho_{\infty}, \rho_{0}$, and $\tau$ correspond respectively to the resistivity after an infinite duration anneal, the resistivity after an infinitesimally short anneal, and the exponential relaxation time for the annealing process. The results at all three annealing temperatures are well described by the function $1-\exp (t / \tau)$, represented by the solid curve in the main figure. The inset to the figure shows the dependence of $\rho^{\mathrm{RT}}$ (in $\mathrm{m} \Omega \mathrm{cm}$ ) on anneal duration (in hours) for a film repeatedly annealed at 203 ${ }^{\circ} \mathrm{C}$. The solid curve in the inset represents a best-fit of the data to the expression $\rho^{\mathrm{RT}}=\rho_{\infty}[1-A \exp (-t / \tau)]$. We ascribe the changes in resistivity to changes in Au grain size and distribution during annealing.

increasing anneal duration for three representative annealing temperatures: $95{ }^{\circ} \mathrm{C}, 145^{\circ} \mathrm{C}$, and $203^{\circ} \mathrm{C}$. In every case, the room-temperature resistivity $\rho^{\mathrm{RT}}$ asymptotically approaches a limiting value $\rho_{\infty}$ with repeated anneals at a fixed annealing temperature. Quantitatively, the time dependence can be empirically fit to the expression

$$
\rho^{\mathrm{RT}}=\rho_{\infty}\left[1-A \exp \left(\frac{-t}{\tau}\right)\right]
$$

where $t$ is the total annealing time, $\rho_{\infty}$ is the asymptotic limit of the room-temperature resistivity for an infinite duration anneal, $A$ is a dimensionless annealing coefficient, and $\tau$ is the relaxation time for the annealing process. The limiting resistivity $\rho_{\infty}$, the extrapolated $t=0$ anneal resistivity $\rho_{0}$, the annealing coefficient $A$, and the annealing time constant $\tau$ are all functions of the annealing temperature $T_{\text {anneal }}$.

As shown in Fig. 2, the annealing time dependence of the room-temperature resistivity $\rho^{\mathrm{RT}}$ can be represented by a "universal" curve when the data is plotted in terms of the fractional change in the room-temperature resistivity $\left(\rho^{\mathrm{RT}}\right.$ $\left.-\rho_{0}\right) /\left(\rho_{\infty}-\rho_{0}\right)$ and the relative anneal duration $t / \tau$, where $\rho_{0}=\rho_{\infty}(1-A)$. Note that this dependence is observed both below and above the critical temperature for $\mathrm{Au}$ assisted $\mathrm{Ge}$ crystallization in these films $\left(135^{\circ} \mathrm{C}\right)$. The absolute depen-

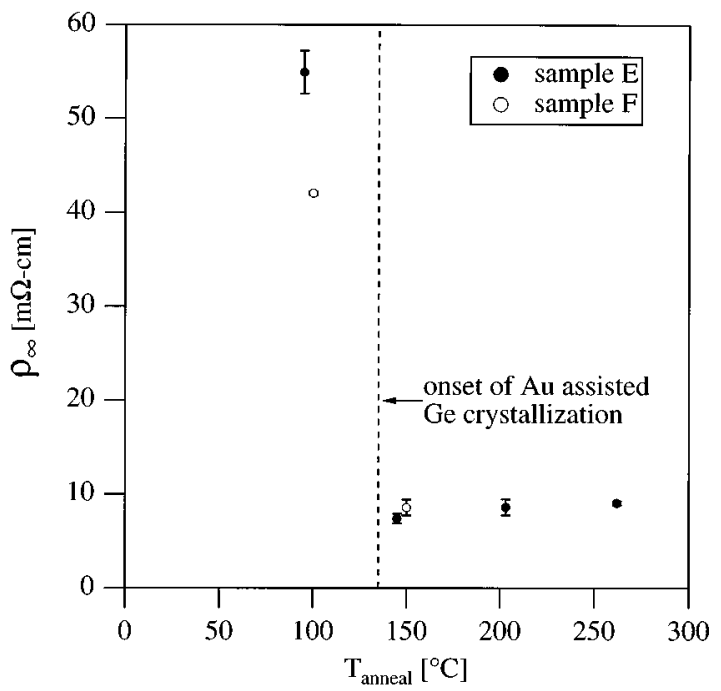

FIG. 3. Dependence of the room-temperature resistivity $\rho^{\mathrm{RT}}$ on annealing temperature for a series of "infinite duration" anneals. For each annealing temperature, the value of $\rho_{\infty}$-determined from a non-linear best fit to Eq. (2) - corresponds to the limiting value of the room-temperature resistivity $\rho^{\mathrm{RT}}$ after repeated annealing. A sharp drop in $\rho_{\infty}$ occurs at $135^{\circ} \mathrm{C}$ due to the onset of Au-assisted Ge crystallization. Samples E and F were sputtered simultaneously but sample E was annealed 3 years after sample F.

dence of the room-temperature resistivity (in $\mathrm{m} \Omega \mathrm{cm}$ ) on anneal duration (in h) for repeated annealing at $203{ }^{\circ} \mathrm{C}$ is shown in the inset to Fig. 2.

The corresponding dependences of $\rho_{\infty}, A$ and $\tau$ on annealing temperature $T_{\text {anneal }}$ are shown in Figs. 3, 4, and 5, respectively. In each case, the error bars represent $\pm 2 \sigma$ for the plotted parameter. The annealing temperature dependence of $\rho_{\infty}$ is in general agreement with the results for the single 30 min anneal previously shown in Fig. 1. More

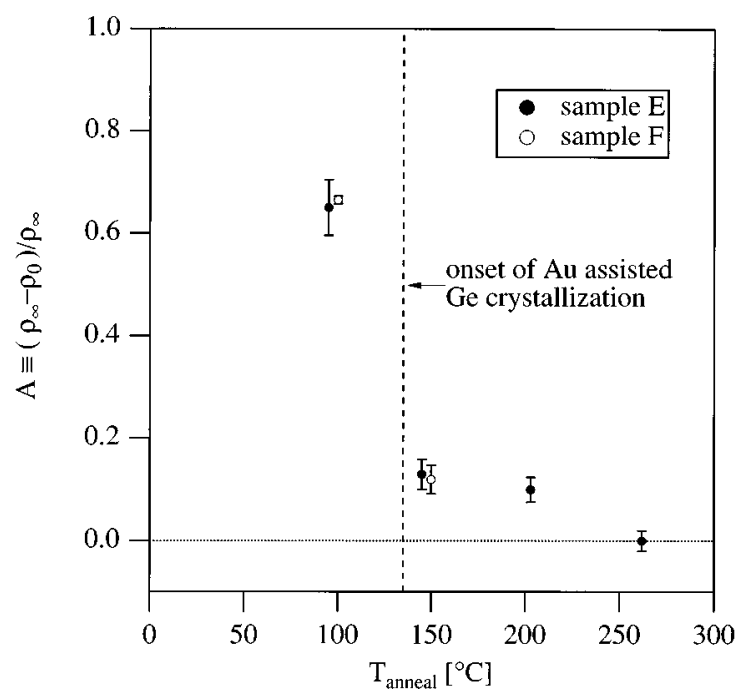

FIG. 4. Fractional change $A$ in the room-temperature resistivity $\rho^{\mathrm{RT}}$ with increasing annealing duration as a function of annealing temperature. $A$ $=1$ corresponds to a $100 \%$ change in $\rho^{\mathrm{RT}}$ between the $t=0$ infinitesimally short and $t=\infty$ infinitely long duration anneal values of $\rho^{\mathrm{RT}}$. For each annealing temperature, the value of $A$ was determined from a nonlinear best fit to Eq. (2). For the commonly reported annealing temperature of $150{ }^{\circ} \mathrm{C}$, $A \approx 0.15$, while for the higher anneal temperature of $265^{\circ} \mathrm{C}, A \approx 0$. 


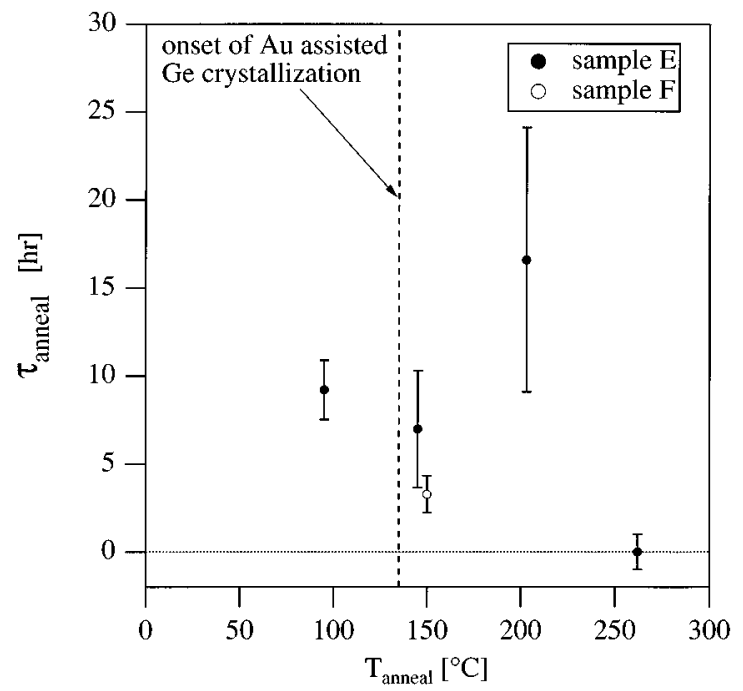

FIG. 5. Variation of the relaxation time $\tau$ with increasing annealing temperature for the exponentially attenuating change in room-temperature resistivity $\rho^{\mathrm{RT}}$ with increasing anneal duration. For each annealing temperature, the value of $\tau$ was determined from a nonlinear best fit to Eq. (2). At the commonly reported annealing temperature of $150{ }^{\circ} \mathrm{C}$, an anneal on the order of tens of hours is needed to prevent drifts in $\rho^{\mathrm{RT}}$ with further heat treatment at or below the annealing temperature. The onset of Au-induced Ge crystallization dramatically reduces the magnitude of $\rho^{\mathrm{RT}}$ but does not significantly change $\tau$, which depends primarily on the rate at which large Au grains grow at the expense of smaller Au grains and the distribution of Au grain sizes.

surprising are the annealing temperature dependences of the annealing coefficient $A$ and relaxation time $\tau$. At a typically reported annealing temperature of $150{ }^{\circ} \mathrm{C}$ - just above the metal-assisted crystallization temperature-the roomtemperature resistivity $\rho^{\mathrm{RT}}$ changes by $15 \%$ and an anneal on the order of tens of hours is needed to render $\rho^{\mathrm{RT}}$ insensitive to further heat treatment at this temperature.

Our results imply that the annealing temperatures of $130-160{ }^{\circ} \mathrm{C}$ and anneal durations of 30-60 min typically reported for $\mathrm{Au}_{0.18} \mathrm{Ge}_{0.82}$ films are inadequate in both temperature and duration. In particular, later heat treatment at or above the annealing temperature-during soldering of leads, for example-will lead to a slowly shifting temperature calibration. The problems associated with inadequate annealing, can be most easily eliminated by annealing at a higher temperature. At an annealing temperature of $265^{\circ} \mathrm{C}$, for example, the room-temperature resistivity $\rho^{\mathrm{RT}}$ appears to have saturated and the annealing coefficient $A$ is reduced to zero (within the error of our measurements), implying that an anneal of $1 \mathrm{~h}$ is already sufficient.

We can gain a rough understanding of this time dependence by modeling the $\mathrm{Au}_{x} \mathrm{Ge}_{1-x}$ film as a distribution of conducting Au grains in a nonconducting amorphous $\mathrm{Ge}$ medium. Under this simplifying assumption, the time dependence of the film resistivity after Ge crystallization will be dominated by changes in the distribution of sizes and separations of Au grains. During annealing, larger Au grains can grow at the expense of smaller grains. ${ }^{10}$ As the grains grow in size, this will lead to a distribution of $\mathrm{Au}$ grains with greater characteristic separation. Since the resistivity can be expected to be an increasing function of average Au grain

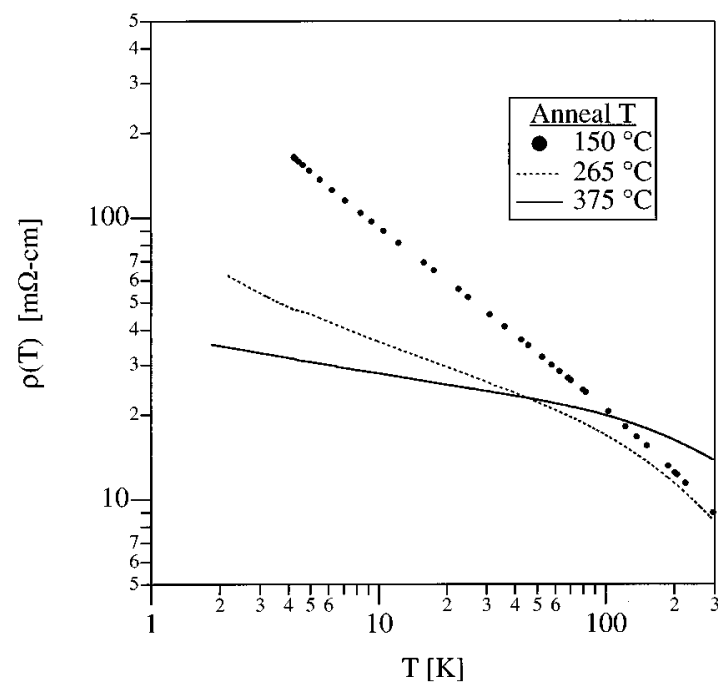

FIG. 6. Log-log graph of the variation of the resistivity $\rho$ with temperature for three fully annealed films annealed at $150^{\circ} \mathrm{C}, 265^{\circ} \mathrm{C}$, and $375^{\circ} \mathrm{C}$, respectively. On this graph, constant sensitivity would correspond to a line of constant slope. The more nearly power law instead of exponential dependence of $\rho(T)$ allows these films to be used as thermometers over an extended temperature range.

separation, ${ }^{11}$ we should find that the resistivity increases with increasing anneal duration at a fixed annealing temperature.

The time dependence of the film resistivity before $\mathrm{Ge}$ crystallization will be more complicated, since the time dependence of the film resistivity will also reflect the depletion of the as-deposited Au layers due to interdiffusion of $\mathrm{Au}$ and Ge. In addition, if the initial Au concentration is excessively high, some undiffused $\mathrm{Au}$ may remain in layers after Ge crystallization. Although these complications are not considered in our simple model, the ability of Eq. (2) to describe the dependence of the room temperature film resistivity $\rho^{\mathrm{RT}}$ on anneal duration for repeated anneals at $95^{\circ} \mathrm{C}$ suggests that $\mathrm{Au}$ grain growth is the dominant contribution to $\rho^{\mathrm{RT}}(t)$ even at relatively low annealing temperatures.

\section{SENSITIVITY}

The temperature dependence of the resistivity is plotted in Fig. 6 on a log-log scale for three otherwise identical films progressively annealed at a series of temperatures up to a maximum of $150{ }^{\circ} \mathrm{C}$ (for $1000 \mathrm{~min}$ ), $265^{\circ} \mathrm{C}$ (for $120 \mathrm{~min}$ ) and $375^{\circ} \mathrm{C}$ (for $30 \mathrm{~min}$ ), respectively. The power law behavior described by Eq. (1) would correspond to a line of constant slope. At first inspection, the sensitivity appears nearly constant at low temperature for all three films but clearly increases with temperature near room temperature. Although the films remain useful as resistive thermometers over the entire temperature range, Eq. (1) must be replaced with a more general expression for nonconstant $\eta$.

We therefore calculated the temperature dependence of the sensitivity $\eta(T)$ by empirically fitting the temperature dependence of the resistivity $\rho(T)$ to the polynomial expression

$$
\ln \rho=\sum_{n=0}^{N} a_{n}(\ln T)^{n}
$$




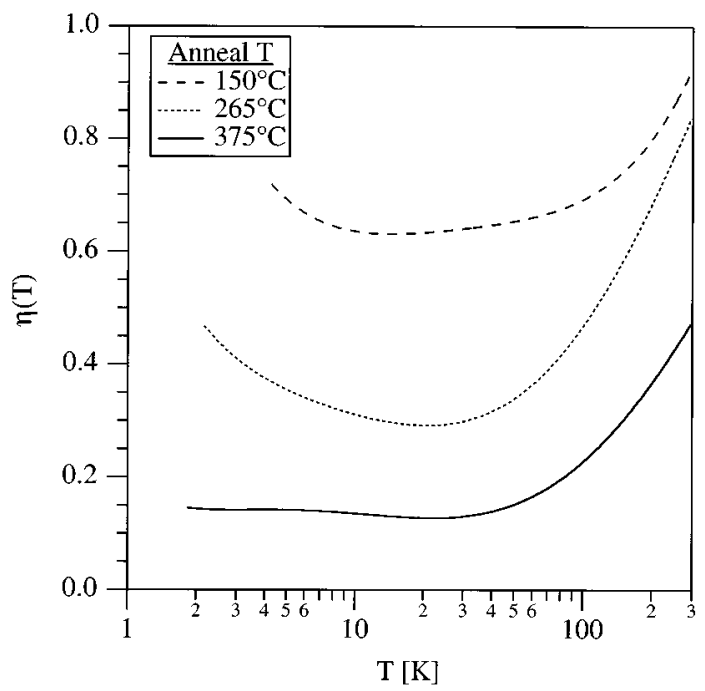

FIG. 7. Semi-log graph of the variation of the sensitivity $\eta$ with temperature for the same three fully annealed films shown in Fig. 6. For all three films, $\eta(T)$ can be fit to the polynomial expansion in $\ln T$ given by Eq. (4) over the entire temperature range. The decreasing sensitivity with increasing annealing temperature illustrates the trade-off between sensitivity and susceptibility to post-anneal heat-treatment-induced shifts in $\rho^{\mathrm{RT}}$.

and differentiating with respect to $\ln T$, yielding the analytic equation

$$
\eta(T) \equiv-\frac{d \ln \rho}{d \ln T}=-\sum_{n=1}^{N} n a_{n}(\ln T)^{n-1}
$$

for the temperature dependence of $\eta$. Equation (3) is often used to fit the temperature dependence of the resistivity of doped Germanium thermometers ${ }^{12}$ - for which the sensitivity is a sharply increasing function of decreasing temperature — and has no implied physical origin.

Given the above analytic expressions for $\rho(T)$ and $\eta(T)$, the calorimetrically important percent change in temperature $\Delta T / T$ corresponding to a measured percent change in resistance $\Delta R / R$ can be easily calculated for small temperature changes using the approximation

$$
\frac{\Delta T}{T} \approx-\frac{1}{\eta}\left(\frac{\Delta R}{R}\right),
$$

where $R=c \rho$ and $c$ is a constant.

In Fig. 7, we show the variation of $\eta$ with temperature for the same films as in Fig. 6. Figure 7 reveals an upturn in $\eta$ below $10-20 \mathrm{~K}$ for the films annealed at $150^{\circ} \mathrm{C}$ and $265^{\circ} \mathrm{C}$ that is not seen in the film annealed at $375^{\circ} \mathrm{C}$. As can also be seen from Fig. 7, however, the sensitivity at any particular temperature is larger for films annealed at lower temperatures. The variation of $\rho(T)$ and $\eta(T)$ at lower temperatures and in magnetic fields is under investigation.

To understand how sensitivity might decrease with increasing annealing temperature, we return to our simplified model of the $\mathrm{Au}_{x} \mathrm{Ge}_{1-x}$ film as a distribution of conducting $\mathrm{Au}$ grains in a non-conducting Ge medium. Consider first how the sensitivity would be expected to vary with increasing Au concentration $x$ if all Au grains were the same radius $r$ and had the same center to center separation $s$. Since
$\mathrm{Au}_{x} \mathrm{Ge}_{1-x}$ is metallic for sufficiently large $x$, we would expect the ratio $r / s$ to increase and the sensitivity $\eta$ to decrease (and ultimately change sign) as $x$ increases from zero, as seen in $\mathrm{Au}_{x}\left(\mathrm{Al}_{2} \mathrm{O}_{3}\right)_{1-x}$ cermets. ${ }^{13}$

Now consider the variation of $r / s$ for our system, in which $x$ is constant but there is a distribution of $r$ and $s$ values. In this case, both $r$ and $s$ are expected to increase, implying that $r / s$ could either increase or decrease. For relatively high (but still nonmetallic) Au concentrations, however, the growth of large Au grains at the expense of small $\mathrm{Au}$ grains will lead to a slower rate of increase in the median value of $s$ than at relatively low Au concentrations. If our model is correct, then, our films have a sufficiently high $\mathrm{Au}$ concentration $x$ that both $s$ and $r / s$ increase with increasing annealing temperature. This also suggests that at sufficiently lower $\mathrm{Au}$ concentrations, an increasing annealing temperature would lead to an increasing sensitivity and at an intermediate Au concentration below $x=0.18$, the dependence of the sensitivity on annealing temperature might be minimized.

As with other resistive cryogenic thermometers (such as doped Germanium or carbon composition resistors) for which the resistance increases with decreasing temperature, there is a trade-off between sensitivity and the temperature range for which temperature measurements can be performed. The lower temperature limit is set by the largest absolute resistance that can be accurately measured without significant self-heating. ${ }^{12}$ For two otherwise identical films with comparable resistivity at higher temperature the resistance will be increasing more sharply with decreasing temperature for the higher sensitivity film, leading to a higher low-temperature limit. For common resistive cryogenic thermometers exhibiting rapidly increasing sensitivity with decreasing temperature, the high temperature limit is usually determined by the minimum acceptable sensitivity for the experiment.

From this perspective one advantage of these $\mathrm{Au}_{x} \mathrm{Ge}_{1-x}$ films is that their more nearly power law instead of exponential dependence on temperature allows measurements to be performed over a greatly extended range of temperatures. For the $\mathrm{Au}_{0.18} \mathrm{Ge}_{0.82}$ films presented here, the tradeoff is no longer between sensitivity and temperature range but instead between sensitivity and susceptibility to calibration shifts due to post-anneal heat treatment.

\section{ACKNOWLEDGMENTS}

One of the authors (N.A.F.) would like to thank the Jean Picker Fellowship (administered by Smith College) for partial support of this research and Y. Katayama of the Materials Science Division of the Electrotechnical Laboratory, Tsukuba, Japan for the loan of his custom-built sputtering system and instruction in its use.

${ }^{1}$ B. W. Dodson, W. L. McMillan, J. Mochel, and R. Dynes, Phys. Rev. Lett. 46, 46 (1981).

${ }^{2}$ D.-M. Zhu and F. Lin, Rev. Sci. Instrum. 64, 2624 (1993).

${ }^{3}$ O. Béthoux, R. Brusetti, J. C. Lasjaunias, and S. Sahling, Cryogenics 35, 447 (1995). 
${ }^{4}$ M. Osofsky, H. Tardy, M. LaMadrid, and J. Mochel, Phys. Rev. B 32, 2101 (1985).

${ }^{5}$ M. Osofsky, J. B. Bieri, M. LaMadrid, W. Contrata, and J. M. Mochel, Phys. Rev. B 38, 12215 (1988).

${ }^{6}$ Z. Tan, S. M. Heald, M. Rapposch, C. E. Bouldin, and J. C. Woicik, Phys. Rev. B 46, 9505 (1992).

${ }^{7}$ F. Oki, Y. Ogawa, and Y. Fujiki, Jpn. J. Appl. Phys. 8, 1056 (1969).

${ }^{8}$ S. Herd, P. Chaudhara, and M. Brodsky, J. Non-Cryst. Solids 7, 309 (1972).
${ }^{9}$ H. Jian-Guo, W. Zi-Qin, and B. Ling-Song, Thin Solid Films 173, 77 (1989).

${ }^{10}$ P. Cotterill and P. Mould, Recrystallization and Grain Growth in Metals (Halsted, New York, 1976), p. 2.

${ }^{11}$ N. Gershenfeldet al., J. E. VanCleve, W. W. Webb, H. E. Fisher, N. A. Fortune, J. S. Brooks, and M. J. Graf, J. Appl. Phys. 64, 4760 (1988).

${ }^{12} \mathrm{~F}$. Pobell, Matter and Methods at Low Temperatures (Springer, Berlin, 1992), p. 222.

${ }^{13}$ B. Abeles, P. Sheng, M. Coutts, and Y. Arie, Adv. Phys. 24, 407 (1975). 